\title{
Applications of atomic force microscopy for the assessment of nanoscale morphological and mechanical properties of bone
}

\author{
Bone 50 (2012) 420-427 \\ Joseph M. Wallace \\ Indiana University-Purdue University at Indianapolis \\ Department of Biomedical Engineering \\ 723 W Michigan St. \\ Indianapolis, IN 46202
}

\begin{abstract}
Scanning probe microscopy (SPM) has been in use for 30 years, and the form of SPM known as atomic force microscopy (AFM) has been around for 25 of those years. AFM has been used to produce high resolution images of a variety of samples ranging from DNA to carbon nanotubes. Type I collagen and many collagen-based tissues (including dentin, tendon, cartilage, skin, fascia, vocal cords, and cornea) have been studied with AFM, but comparatively few studies of bone have been undertaken. The purpose of this review is to introduce the general principles of AFM operation, demonstrate what AFM has been used for in bone research, and discuss the new directions that this technique can take the study of bone at the nanoscale.
\end{abstract}

\section{Introduction}

As a tissue and organ, bone has elegantly evolved to serve vital structural and metabolic roles in the body. Like many tissues, bone is hierarchical [1], having structural elements that exist independently at length scales ranging over 9-10 orders of magnitude from the molecular level to the organ level. At the nanoscale, bone is primarily a two-phase composite material composed of a flexible organic matrix (approximately 90\% Type I collagen) which is impregnated with and surrounded by a stiffer, stronger, reinforcing apatite mineral phase. The combination of strength, stiffness and toughness that are mechanical characteristics of bone as a tissue are initially derived from the intimate interaction between these nanoscale constituents of vastly differing mechanical properties [2]. Bone is unique in that it delicately balances strength and toughness with minimal mass while contending with the constant structural and metabolic demands of the body. Despite 
the importance of skeletal health to overall health, bone as a material is still only partially understood. Most notably, there is at best an incomplete understanding of how the nanoscale properties of collagen and mineral, and their interaction with one another, produce the mechanically-competent structural organ that exists at larger length scales [3] and [4]. Additionally, it is unknown how perturbations to the individual constituents (such as those that may occur with disease, alteration in nutrition, trauma or in response to mechanical stimulation) change bone's mechanical behavior. It is therefore imperative for researchers to develop accurate quantitative methods to assess the nanoscale properties of normal bone in order to develop an understanding of the composition, assembly and organization of the fundamental building blocks of this amazing tissue.

Bone researchers come from a variety of backgrounds ranging from the archetypal biologist to the classic engineer. As such, we have borrowed an array of analytical techniques from other branches of science to ask questions related to bone. Several of the more successful techniques used to assess the compositional quality of bone include X-Ray Diffraction [5] (XRD), Fourier Transform Infrared [6] and [7] (FTIR) Spectroscopy and Raman Spectroscopy [8] and [9]. These methods have yielded a wealth of information regarding the chemical state of bone. Other $\mathrm{x}$-raybased methods such as Small Angle X-Ray Scattering (SAXS) and Wide Angle X-Ray Scattering (WAXS) have been used to analyze mineral crystal thickness and larger-scale matrix organization [10] and [11]. However, these techniques lack the ability to directly answer questions about the nanoscale assembly and organization of collagen and mineral in bone. To probe the nanoscale and produce images of collagen and mineral, researchers have traditionally relied on methods such as transmission electron microscopy (TEM) and scanning electron microscopy (SEM). Without question, these methods can produce high-resolution images of the collagen and mineral components of the bone matrix. However, both techniques have significant limitations. To perform TEM in mineralized tissues, the sample is typically fixed and fully dehydrated, embedded in a hard resin, cut with a diamond blade to produce ultra-thin sections and imaged under vacuum [12]. For SEM, samples are dehydrated and coated with either carbon or gold to increase contrast, then imaged under vacuum [10] and [13]. The combination of harsh sample preparation and imaging conditions may induce artifacts and ultimately reduce the accuracy of conclusions drawn from the data. A relative newcomer to the scene, atomic force 
microscopy (AFM), may revolutionize the study of the nanoscale properties of bone and other Type I collagen-based tissues. As a technique, AFM is relatively inexpensive and simple to perform. It is a high-resolution imaging modality which is less-destructive than either TEM or SEM. Samples imaged using AFM can remain intact and can be imaged in air or fluid, at room temperature or under culture conditions, implying that measured properties are characteristics of the sample and less likely artifacts of processing or imaging [14] and [15]. In addition, the AFM probe can be used to extract nanoscale mechanical properties (both in terms of the forces and physical size of material that is probed) by scratching, indenting, pushing or pulling on the sample. This review will focus on the current state of knowledge of AFM and its application to the imaging and nanoscale manipulation of bone.

\section{A brief history of atomic force microscopy}

As opposed to traditional microscopes which focus light or electrons on a sample to produce an image, AFM works by dragging a sharpened probe over the surface and using interactions between the surface and probe to build up a map of the sample's topography. The easiest real world analogy of the AFM is the phonograph or record player. An early instrument that worked by dragging a sharpened probe over a surface was developed by Gustav Schmalz in 1929 [16]. Known as the stylus profiler, it operated by dragging a stylus mounted on the end of a rigid bar across a sample to produce a height profile. Due to bending caused as the "probe" met lateral resistance from surface features, the resolution and fidelity of the resulting image were suspect. A refinement came in 1955 when Becker oscillated a probe to reduce surface drag [17]. In 1972, the first major step forward came with Russell Young's Topografiner [18]. Young took advantage of piezoelectric ceramic elements which expand and contract in a highly controlled manner with an applied electric potential. A metal probe was mounted onto a piezoelectric element which moved the probe in the vertical direction to control the probe-sample separation distance. An electron field developed between the probe and sample, and varied as a function of distance between the two. Therefore, a feedback system was used to monitor the electron field and keep this distance at a constant value as the probe was scanned across a surface. The limiting factor governing the resolution of the Topografiner was the system's vibration, a problem that was finally tackled by Binnig and Rohrer at IBM in 1982 with the invention of the first Scanning Tunnelling Microscope (STM) [19]. By improving the vibration isolation of the system, they 
were able to monitor electron tunnelling rather than electron field emissions. This tunnelling current is much more sensitive to tip-sample interactions, so the probe could be kept very close to the surface. The result was a small device that produced atomic resolution images, a feat that earned Binnig and Rohrer the Nobel Prize in Physics in 1986. Recognizing that STM was limited to electrically-conductive materials, Binnig et al. replaced the conductive wire probe with a diamond glued to a strip of gold, producing the first Atomic Force Microscope in 1986 [20]. Over the next 25 years, modifications to basic operation modes and other hardware and software improvements have been made. As many have reviewed these topics, the reader is referred to two recent books for more detailed information [15] and [21]. 


\section{Basic AFM operation}

The operation of AFM for imaging purposes is governed by three basic components (Fig. 1). Piezoelectric elements, which create mechanical movement under an applied electric potential, are used to accurately move the AFM probe independently in three dimensions. As the probe is raster-scanned across the sample in the $\mathrm{x}-\mathrm{y}$ direction (or, conversely, the sample is moved under the probe), the force of the interaction between the sample and the probe is measured by a force transducer, typically the cantilever on which the probe is mounted. Finally, a feedback control system is used to maintain a desired force between the probe and the sample. When the measured force it larger or smaller than this set point, a voltage is applied to the z-piezo to move the probe away from or towards the surface, bringing the force back to the set point. Although these general concepts seem simple, their integration into a working system is complex and the reader is referred to another source for more details [15].

To understand the operation of an AFM at a basic level, one must recognize that interactions are occurring between the probe and sample at the molecular level. A combination of interactive forces including magnetic, electrostatic and capillary forces is important depending on the separation distance, but van der Waals forces usually dominate at small distances. Fig. 2 shows the van der Waals potential energy curve which describes these interactions as the probe is brought close to the sample's surface. Initially, at large distances (the right of the image), weak attractive interactions exist between atoms at the surface of the sample and those at the apex of the probe. As the distance decreases, this attractive force increases until the electron clouds of the atoms begin to interact and electrostatically repel each other. With further decrease in the interatomic separation distance, this repulsive force weakens the overall attraction until a net force of zero is reached. At this point, the distance between the atoms is on the order of $2-3 \AA$, about the length of a chemical bond. With further decrease in separation, the interactive forces become positive (repulsive) and the atoms are said to be in "contact." Note that in the "contact" region, the slope of the curve is quite steep meaning that the repulsive van der Waals forces balance almost any attempt to push the atoms closer together. In the case of AFM, when the cantilever attempts to push the probe against the sample, the cantilever instead deflects. Since the cantilever acts like a linear spring and deflects according to Hooke's law (i.e. $\mathrm{F}=-\mathrm{kx}$ where $\mathrm{k}$ is 
the spring constant and $\mathrm{x}$ is the deflection), the cantilever can be used as a sensitive force transducer.

\section{AFM-based imaging}

There are many operating modes for AFM, but most investigations generally operate using one of two main feedback modes. In Contact Mode, the probe is in physical contact with the surface at all times, operating in the repulsive regime. While scanning, the cantilever deflects up or down to accommodate topography under the probe while the feedback loop maintains a constant userdefined cantilever deflection (i.e. imaging force) by vertically moving the sample using the Zpiezoelectric actuator. This movement of the piezo at each pixel location generates a height profile image of the surface (Fig. 3F). This method of operation is good for wet or dry imaging and can produce atomic resolution images, but the probe is always touching the sample. Because of lateral forces that develop as the probe moves across the surface, damage to both the sample (especially soft samples) and the probe are possible.

The second main operating mode, which alleviates some of this problem, is known as Intermittent Contact or Tapping Mode. In this mode, the cantilever is oscillated very near its resonance frequency (in the 10's to 100's of $\mathrm{kHz}$ for many commercially available probes) as the probe is scanned across the surface. The user-defined amplitude of oscillation is typically set between 10 and $100 \mathrm{~nm}$, and the probe "taps” the surface at the bottom of the bounce. As the probe interacts with topographic features, the oscillation amplitude changes and the feedback loop moves the sample up or down as described above to maintain the amplitude set point. Since the probe is in contact with the surface for a small fraction of its tapping period, lateral forces are reduced. In some cases, this can lead to higher lateral resolution. However, a drawback of this method is that imaging in fluid can be challenging. The cantilever must be "tuned" at its resonance frequency, which is straightforward in air. In fluid, the resonance frequency is usually altered and many spurious peaks exist, making it difficult to properly tune the probe.

A recently implemented imaging mode, PeakForce Tapping, oscillates the probe at $1 \mathrm{kHz}$, far below the resonance frequency of most commercially available probes. Because of this relatively slow rate, a force curve (Fig. 4) is generated with each tap and the maximum (peak) force of the 
tap is used as the feedback signal. Because force is directly controlled, it can be minimized to reduce sample damage and tip wear. In addition, because the cantilever is not tuned, imaging in fluid is much less challenging.

\section{AFM-based indentation}

Given that the cantilever is a sensitive force transducer, the cantilever/probe combination can be used to push on a sample to extract useful elastic mechanical properties. The probe is pushed into the surface to a known load or displacement, during which time a force-displacement curve is collected (Fig. 4). This curve can then be analyzed using one of many contact mechanics approaches. The most common method, the Hertz model, describes the indentation of an infinitely hard spherical indenter (the probe tip) on an elastic cylinder (the sample) using a force that is normal to the surface. A weakness of using AFM to indent a sample is that the probe interacts with the surface at an angle. Therefore, the force applied during an indentation has both a normal and lateral component. Since the lateral component is small in comparison to the normal component, it is usually disregarded. To obtain the elastic modulus using the Hertz model, the slope of the withdrawal portion of the force curve is fit to the equation:

$\mathrm{E}=\frac{3 \mathrm{~F}\left(1-v^{2}\right)}{4 r^{1 / 2} \delta^{3 / 2}}$

In this equation, $\mathrm{F}$ is the applied load, $v$ is Poisson's ratio for the sample, $\mathrm{r}$ is the radius of curvature of the AFM probe and $\delta$ is the indentation depth. In addition to elastic modulus, maximum (peak) force, deformation, adhesion force and energy dissipation can be extracted from the raw data. A strength of AFM-based indentations, in comparison to an instrumented nanoindentation system, is the force and deformation range. AFM is a true nanoscale approach, where the forces and deformations remain "nano" in scale. In a traditional "nano"indentation test, forces of greater than $1 \mathrm{mN}$ are not uncommon, leading to large deformations on the order of $1 \mu \mathrm{m}$ (please see [22] for a thorough review of the topic of nanoindentation studies in bone). This level of indentation force can lead to local yielding and permanent deformation of the sample. Two recent reviews cover the topic of AFM-based indentation theory in detail [23] and [24].

\section{AFM applications in bone research}


In the process of researching the literature for this review, approximately 150 peer-reviewed journal articles were discovered that addressed the application of AMF-based techniques to the investigation of bone, dentin, tendon and other collagen-based tissues since the first study in 1989 (Fig. 5). Of these, 25\% relate to bone. Although much can be learned from AFM imaging studies in other collagen-based tissues, this review will focus on those papers related to advancements in the study of bone.

In its infancy, Scanning Probe Microscopy (SPM, includes both STM and AFM) was used almost for its novelty, as most researchers still heavily relied on SEM and TEM (and in fact, many still so). The first SPM study in collagen came in 1989 when investigators used STM to study surface features on purified collagen proteins [25]. This investigation was followed by a few additional STM studies of collagen and by 1992, the first article on AFM in collagen was published [26]. Although the first investigation in bone came in 1993 [27], and was followed 2 years later by a similar study by the same group [28], the next independent study was not published until 2001. Since then, there was a peak in 2005-2006 that has since dropped off with only a few papers per year. The majority of AFM papers devoted to bone are primarily imaging studies looking at one of the two major components which make up the bone extracellular matrix (ECM).

\section{Investigating bone cell activity and canilicular structure}

In its debut application to bone research in 1993, AFM was used to measure the depth, volume and surface topography of Howship's lacunae left behind by osteoclasts cultured on slices of sperm whale dentin [27]. As was the case with many early AFM investigations, this study was largely qualitative, although an average resorption pit depth of $3.7 \pm 0.73 \mu \mathrm{m}$ and volumes between $473 \mu \mathrm{m} 3$ and $1432 \mu \mathrm{m} 3$ were reported. Other investigations of osteoclast activity followed [28], and some attempts were made to assess the underlying collagen structure left behind after mineral dissolution [29] and [30]. However, detailed analyses of bone collagen morphology were not presented. A more recent investigation used AFM to monitor collagen deposition and the progression of mineralization in the osteocyte-like cell line MLO-A5 in culture [31]. AFM has also been used to analyze the distribution and dimensions of the lacunarcanilicular network in bone [32], [33] and [34]. These studies put the average canilicular 
diameter at around 400-500 nm, larger than values typically reported from TEM studies. In addition, one study found that the diameter and distribution of canaliculi are constant throughout individual osteons and that globular structures often coat their surfaces [34].

\section{Studies of bone mineral}

By coincidence or by design, researchers studying bone cell activity bypassed one of the main difficulties of nanoscale analysis in bone: bone is mineralized. Some work with AFM has been performed specifically to analyze the mineral in bone. In two studies in young and old bovine bone, mineralites were freed of their organic counterparts, and their dimensions were measured. In older samples, the mineral was thin and plate-like, with dimensions of $12 \mathrm{~nm} \times 10 \mathrm{~nm} \times 0.61$ $\mathrm{nm}$ [35]. Under similar conditions in younger animals, the mineralites were thicker plates with dimensions of $9 \mathrm{~nm} \times 6 \mathrm{~nm} \times 2 \mathrm{~nm}$ [36]. In both cases, these dimensions are much smaller (at least in terms of length and width) than typically cited values from TEM [37]. However, TEM values often have a large range. Other studies of the state of mineral in situ have shown a variety of morphologies and sizes for mineral crystals [38], [39] and [40]. Although more physiologically relevant, these studies are complicated by the topography of the underlying collagen matrix which makes interpretation challenging.

\section{Investigating collagen in bone}

In order to observe structures in bone other than a blanket of mineral covering collagen fibrils, some amount of demineralization is necessary (Fig. 3). A variety of acid types (citric acid [41], phosphoric acid [40] and [42], formic acid [43]) and concentrations have been used to etch the surface of mineralized tissues, with varying degrees of success. Chelating agents such as Ethylenediaminetetraacetic Acid (EDTA) have also been used successfully. As opposed to acid etching, demineralization using EDTA is a slower and "gentler" process, and is often used in bone research to fully demineralize a sample. One limitation of demineralization of any kind is that although the collagen is exposed, the effects of the treatment on collagen structure are not known.

Following demineralization, a variety of properties can be analyzed and quantified. Baranauskas et al. used AFM to assess the spatial organization of newly formed bone following a surgically- 
induced injury [44] and [45]. The most commonly measured property of the collagen network in bone is fibril diameter. In some of the first studies of bone using AFM, fibril diameters were reported with wide variability from crude surface measurements, ranging from $110 \pm 10 \mathrm{~nm}$ [39] to $400 \mathrm{~nm}$ [46]. Refinements in imaging and measurement technique have produced more consistent values for fibril diameter below $100 \mathrm{~nm}(83.2 \pm 5.4 \mathrm{~nm}$ [43] and $62.2 \pm 7.0 \mathrm{~nm}$ [30]). Caution must be taken when interpreting and reporting diameters measured from fibrils in bone. The fibril being measured may not be fully demineralized and, therefore, the diameter likely includes contributions from both intrafibrillar and extrafibrillar mineral. A more significant problem has to do with fibril packing. As noted by Ge et al. [43], fibrils are often packed together into a fibril array. Since the entire width of the fibril may not be exposed, any measurement of diameter could be flawed.

Another characteristic of collagen that lends itself to investigation by AFM is the D-period spacing of the fibril. The D-period of collagen creates an oscillating surface topography with a characteristic axial repeat distance of $67 \mathrm{~nm}$. AFM measurements of the D-period in bone collagen have yielded values which were in line with this theoretical value at $67 \pm 2 \mathrm{~nm}$ [39], $66.5 \pm 1.4 \mathrm{~nm}$ [30] and $66.6 \pm 3.8 \mathrm{~nm}$ [43]. The authors of these papers were content with the fact that the spacing was observable, and the measured values were close to the theoretical value. A study in dentin in 2002 showed that the D-period may be present as a distribution of values, although the distribution was not thoroughly studied [41]. This observation, however, prompted our lab to pursue the distribution further. By taking advantage of the periodic nature of the Dspacing, a 2D Fast Fourier Transform (FFT) was used to accurately extract the D-spacing of individual collagen fibrils. Using this technique, we found that regardless of cellular origin or anatomical location, normal Type I collagen-based tissues (including bone) contain a distribution of D-periodic spacings [47]. In bone samples from male and female mice, statistically indistinguishable distributions were observed. The presence of these distributions suggested to us that this metric may represent a means to observe changes that occur with disease in bone at the nanoscale. To date, we have shown that with ovariectomy-induced estrogen depletion in sheep [48] and Osteogenesis Imperfecta in mice [49], there are statistically significant shifts in the Dperiod distributions. Additional work is necessary to tie these changes in collagen morphology to alterations in mechanical function. 


\section{Important research impacts of note}

One research group has made many important contributions to the AFM literature as it pertains to bone, and a review of the topic would be incomplete without discussing some of their work. Paul Hansma's laboratory has published at least 9 individual papers related to bone applications for AFM. The first of these studies was published in Letters to Nature in 2001 [50] and although relatively short, it described using AFM as a tool to push and pull on bone samples to extract meaningful mechanical information. This paper introduced the term "sacrificial bond” referring to bonds within or between collagen fibrils in bone that rupture to dissipate energy as a toughening mechanism. This term is still completely unknown in other arenas such as chemistry and biophysics, despite the fact that the use of AFM to study binding forces in many materials predates this paper by many years. This paper in 2001 was followed by others which further investigated the idea of sacrificial bonds and hidden length scales within the non-collagenous proteins that are likely responsible for their formation [51], [52] and [53]. Although other papers from the group are largely qualitative, they are no less important as they investigate the exposed fracture surfaces of individual trabeculae in bovine and human samples [54], [55], [56], [57] and [58].

\section{Mechanical characterizations in bone}

Despite the fact that AFM can operate under almost any imaging conditions, nearly every study mentioned to this point was performed on dried samples in air. For reasons previously noted, imaging in air is considerably less challenging than imaging in fluid. For measurements of morphology alone, imaging in dry conditions may be acceptable. However, when attempting to mechanically characterize a collagen-based sample, fluid conditions must be used as bone is viscoelastic and mechanical properties change with dehydration. Preliminary data from our lab highlights the importance of hydration when working with collagen-based samples. The impacts of hydration were analyzed in Type I collagen fibrils isolated from the mouse tail tendon. Samples were imaged and mechanically indented in air and in phosphate buffered saline. These data suggest that mechanical properties of collagen fibrils are highly dependent on the presence of fluid, but the D-spacing of the fibrils is not. In fact, there was a three-order of magnitude 
increase in elastic modulus in the dry versus wet state $(1520 \pm 860 \mathrm{MPa}$ in dry vs. $3.0 \pm 0.6 \mathrm{MPa}$ in wet), a finding similar to other AFM indentation data from tendon samples [59].

Only four papers that analyzed bone in fluid using AFM have been published. The first was a study from the Hansma group in 2007 and although they did present an image in fluid, nothing was quantified [57]. A second paper in 2007 performed mechanical characterization on hydrated samples of osteonal bone, but the samples were imaged in air [60]. The most recent study, published in August of 2011, was also interested in the mechanical characterization of cortical bone [61]. However, a direct comparison between wet and dry conditions was not included. As this field moves forward, characterization of the effects of dehydration on nanoscale morphology and mechanics of bone are needed.

\section{A summary of AFM's strengths and weaknesses}

AFM as a technique has many strengths, including that it is a small and easy to use instrument. AFM works in fluids and physiological conditions (e.g. $37^{\circ} \mathrm{C}, 5 \% \mathrm{CO} 2$ ), it is relatively nondestructive to samples (e.g. no fixing, dehydrating, staining, freezing or coating), it produces images in 3 dimensions with high resolution ( $<0.1 \mathrm{~nm}$ in the z-direction, typically $<5 \mathrm{~nm}$ in $\mathrm{x}$ and y), and it can be used to mechanically probe samples. Finally, AFM is a true nanoscale technique, producing both deformations and forces that remain in the nano regime.

As with any technique, there are limitations when using AFM. Piezo actuators have a limited range in the z-direction, meaning that sample features typically must be less than $20 \mu \mathrm{m}$ tall (or deep). In addition, since the probe is "feeling" the surface, there is the possibility that the shape of the probe is convoluted into the resulting image [15]. This problem is made worse as a tip is dulled or damaged with imaging. Tip convolution, along with other imaging artifacts, can make it difficult to interpret images, so care must be taken to ensure that what is seen is repeatable. As noted in the indentation section, the probe interacts with the surface at an angle. Therefore, the force applied during an indentation has both a normal and lateral component. In bone research, much can be learned about the state of both collagen and mineral. Exposing the collagen requires some amount of demineralization, and the impacts of this on collagen structure and stability are 
not fully understood. Despite these limitations, there is much that this technique can do to push the bone research field forward.

\section{Future directions and concluding remarks}

The purpose of this review was to introduce the principles of Atomic Force Microscopy, and how AFM can be used to analyze the structure and function of bone at the nanoscale. Although AFM has been in use for 25 years, it is still in its infancy in application to bone. As noted above, one important direction for the future of AFM in bone research needs to be an increase in our understanding of the nanoscale impacts of dehydration. Another question that may be pursued by pushing AFM techniques to their current limits is to understand the interaction between collagen and mineral at the nanoscale. For example, a recent study demonstrated the imaging of an individual trabecula while under a bending load [58]. It may be possible to study bone under load conditions to understand deformation mechanisms, or the nanoscale development of damage. Another direction is the study of the loading-induced piezoelectric charges produced in bone [62] and [63]. Using a form of AFM known as piezoresponse force microscopy, Minary-Jolandan and Yu demonstrated piezoelectric heterogeneity in bone collagen that corresponds with the D-period spacing. This finding further supports the notion of a role of piezoelectricity in mechanotransduction in bone. One final new direction that AFM is being pushed is in the combination of AFM imaging, force measurements and optical fluorescent microscopy. AFM provide high resolution 3D images of surface features and the ability to characterize biologicallyrelevant forces, while optical methods provide chemical and functional capabilities and the ability to look below the surface. When coupled, they provide the ability to directly and simultaneously correlate optically-viewed features (e.g. fluorescently-tagged structures) with surface topography and mechanics [64]. In addition, AFM can detect magnetic, electrical and piezoelectric forces, and the tip can be functionalized to interact with surface receptors on cells. With the development and implementation of new hardware, these and other exciting new research directions can be tackled. 


\section{Acknowledgments}

The author would like to thank David Burr from the Indiana University School of Medicine for his help in revising the content included in this review. 


\section{References}

[1] Lakes R. Materials with structural hierarchy. Nature 1993;361(6412):511-5.

[2] Ritchie RO, Buehler MJ, Hansma P. Plasticity and toughness in bone. Phys Today 2009;62:41-7.

[3] Fratzl P, Gupta HS, Paschalis EP, Roschger P. Structure and mechanical quality of the collagen-mineral nano-composite in bone. J Mater Chem 2004;14(14): 2115-23.

[4] Gupta HS, Wagermaier W, Zickler GA, Raz-Ben Aroush D, Funari SS, Roschger P, et al. Nanoscale deformation mechanisms in bone. Nano Lett 2005;5(10): 2108-11.

[5] Silverman SR, Fuyat RK, Weiser JD. Quantitative determination of calcite associated with carbonate-bearing apatites. Am Mineral 1952;37:211-22.

[6] Paschalis EP, Verdelis K, Doty SB, Boskey AL, Mendelsohn R, Yamauchi M. Spectroscopic characterization of collagen cross-links in bone. J Bone Miner Res 2001;16 (10):1821-8.

[7] Mendelsohn R, Hassankhani A, DiCarlo E, Boskey A. FT-IR microscopy of endochondral ossification at 20 spatial resolution. Calcif Tissue Int 1989;44(1):20-4.

[8] Timlin JA, Carden A, Morris MD. Chemical microstructure of cortical bone probed by Raman transects. Appl Spectrosc 1999;53(11):1429-35.

[9] Wallace JM, Golcuk K, Morris MD, Kohn DH. Inbred strain-specific response to biglycan deficiency in the cortical bone of C57BL6/129 and C3H/He mice. J Bone Miner Res 2009;24(6):1002-12.

[10] Thurner PJ, Chen CG, Ionova-Martin S, Sun L, Harman A, Porter A, et al. Osteopontin deficiency increases bone fragility but preserves bone mass. Bone 2010;46: 1564-73.

[11] Gupta HS, Seto J, Wagermaier W, Zaslansky P, Boesecke P, Fratzl P. Cooperative deformation of mineral and collagen in bone at the nanoscale. Proc Natl Acad Sci U S A 2006;103(47):17741-6.

[12] Giraud-Guille MM. Twisted plywood architecture of collagen fibrils in human compact bone osteons. Calcif Tissue Int 1988;42(3):167-80.

[13] Weiner S, Arad T, Sabanay I, Traub W. Rotated plywood structure of primary lamellar bone in the rat: orientations of the collagen fibril arrays. Bone 1997;20 (6):509-14.

[14] Morris VJ, Kirby AR, Gunning AP. Atomic force microscopy for biologists. Norwich, UK: Imperial College Press; 1999.

[15] Eaton P, West P. Atomic Force Microscopy. Oxford Univ Press; 2010. 
[16] Schmalz G. Uber Glatte und Ebenheit als physikalisches und physiologishes Problem; 1929.

[17] Becker H. Apparatus for measuring surface irregularities; 1955.

[18] Young R, Ward J, Scire F. The topografiner: an instrument for measuring surface microtopography. Rev Sci Instrum 1972;43(7):999-1011.

[19] Binnig G, Rohrer H, Gerber C, Weibel E. Surface studies by scanning tunneling microscopy. Phys Rev Lett 1982;49(1):57-61.

[20] Binnig G, Quate CF, Gerber C. Atomic force microscope. Phys Rev Lett 1986;56(9): 930-3.

[21] Braga PC, Ricci D. Atomic force microscopy: biomedical methods and applications.

Humana Pr Inc.; 2004

[22] Thurner PJ. Atomic force microscopy and indentation force measurement of bone.

Wiley Interdiscip Rev Nanomed Nanobiotechnol 2009;1(6):624-49.

[23] Lin DC, Dimitriadis EK, Horkay F. Robust strategies for automated AFM force curve analysis-I. Non-adhesive indentation of soft, inhomogeneous materials. J Biomech Eng 2007;129:430-40.

[24] Butt HJ, Cappella B, Kappl M. Force measurements with the atomic force microscope: technique, interpretation and applications. Surf Sci Rep 2005;59(1-6): 1-152.

[25] Hameroff SR, Simic Krstic J, Kelley MF, Voelker MA, He JD, Dereniak EL, et al. Scanning tunneling microscopy of biopolymers: conditions for microtubule stabilization. J Vac Sci Technol A Vac Surf Films 1989;7(4):2890-4.

[26] Chernoff EAG, Chernoff DA. Atomic force microscope images of collagen fibers.

J Vac Sci Technol A Vac Surf Films 1992;10(4):596-9.

[27] Sasaki T, Debari K, Hasemi M. Measurement of Howship's resorption lacunae by a scanning probe microscope system. J Electron Microsc 1993;42(5):356-9.

[28] Debari K, Sasaki T, Udagawa N, Rifkin B. An ultrastructural evaluation of the effects of cysteine-proteinase inhibitors on osteoclastic resorptive functions. Calcif Tissue Int 1995;56(6):566-70.

[29] Hassenkam T, Jorgensen HL, Lauritzen JB. Mapping the imprint of bone remodeling by atomic force microscopy. Anat Rec A Discov Mol Cell Evol Biol 2006;288 (10):1087-94. 
[30] Bozec L, Groot J, Odlyha M, Nicholls B, Nesbitt S, Flanagan A, et al. Atomic force microscopy of collagen structure in bone and dentine revealed by osteoclastic resorption. Ultramicroscopy 2005;105(1-4):79-89.

[31] Barragan-Adjemian C, Nicolella D, Dusevich V, Dallas M, Eick J, Bonewald L. Mechanism by which MLO-A5 late osteoblasts/early osteocytes mineralize in culture: similarities with mineralization of lamellar bone. Calcif Tissue Int 2006;79(5):340-53.

[32] Reilly GC, Knapp HF, Stemmer A, Niederer P, Knothe Tate ML. Investigation of the morphology of the lacunocanalicular system of cortical bone using atomic force microscopy. Ann Biomed Eng 2001;29(12):1074-81.

[33] Knapp HF, Stemmer A, Reilly GC, Niederer P, Tate MLK. Development of preparation methods for and insights obtained from atomic force microscopy of fluid spaces in cortical bone. Scanning 2002;24(1):25-33.

[34] Lin Y, Xu S. AFM analysis of the lacunar canalicular network in demineralized compact bone. J Microsc 2010;241(3):291-302.

[35] Eppell SJ, Tong W, Katz JL, Kuhn L, Glimcher MJ. Shape and size of isolated bone mineralites measured using atomic force microscopy. J Orthop Res 2001;19(6): 1027-34.

[36] Tong W, Glimcher M, Katz J, Kuhn L, Eppell S. Size and shape of mineralites in young bovine bone measured by atomic force microscopy. Calcif Tissue Int 2003;72(5): 592-8.

[37] Weiner S, Traub W. Bone structure: from angstroms to microns. FASEB J 1992;6(3): 87985.

[38] Kindt JH, Fantner GE, Thurner PJ, Schitter G, Hansma PK, editors. A new technique for imaging Mineralized Fibrils on Bovine Trabecular Bone Fracture Surfaces by Atomic Force Microscopy 2005: Warrendale, Pa.; Materials Research Society; 1999.

[39] Sasaki N, Tagami A, Goto T, Taniguchi M, Nakata M, Hikichi K. Atomic force microscopic studies on the structure of bovine femoral cortical bone at the collagen fibril-mineral level. J Mater Sci Mater Med 2002;13(3):333-7.

[40] Mineralised tissues as nanomaterials: analysis by atomic force microscopy. In: Bozec L, de Groot J, Odlyha M, Nicholls B, Horton MA, editors. IEEE Proceedings - Nanobiotechnology; 2005.

[41] Habelitz S, Balooch M, Marshall SJ, Balooch G, Marshall Jr GW. In situ atomic force microscopy of partially demineralized human dentin collagen fibrils. J Struct Biol 2002;138(3):227-36.

[42] El Feninat F, Ellis TH, Sacher E, Stangel I. Moisture-dependent renaturation of collagen in phosphoric acid etched human dentin. J Biomed Mater Res 1998;42(4): 549-53. 
[43] Ge J, Cui FZ, Wang X, Wang Y. New evidence of surface mineralization of collagen fibrils in wild type zebrafish skeleton by AFM and TEM. Mater Sci Eng C 2007;27(1): 46-50.

[44] Baranauskas V, Garavello-Freitas I, Jingguo Z, Cruz-Höfling MA. Observation of the bone matrix structure of intact and regenerative zones of tibias by atomic force microscopy. J Vac Sci Technol A Vac Surf Films 2001;19(4):1042-5

[45] Baranauskas V, Garavello I, Jingguo Z, Cruz-Höfling MA. Analyses of regenerative bone matrix of rat tibia after laser photo-excitation by SEM and AFM. Appl Surf Sci 2005;248(14):492-8.

[46] Thalhammer S, Heckl WM, Zink A, Nerlich AG. Atomic force microscopy for high resolution imaging of collagen fibrils - a new technique to investigate collagen structure in historic bone tissues. J Archaeol Sci 2001;28(10):1061-8.

[47] Wallace JM, Chen QS, Fang M, Erickson B, Orr BG, Holl MMB. Type I collagen exists as a distribution of nanoscale morphologies in teeth, bones, and tendons. Langmuir 2010;26(10):7349-54.

[48] Wallace JM, Erickson B, Les CM, Orr BG, Holl MMB. Distribution of type I collagen morphologies in bone: relation to estrogen depletion. Bone 2010;46(5):1349-54.

[49] Wallace JM, Orr BG, Marini JC, Banaszak Holl MM. Nanoscale morphology of type I collagen is altered in the Brtl mouse model of osteogenesis imperfecta. J Struct Biol 2011;173:146-52.

[50] Thompson JB, Kindt JH, Drake B, Hansma HG, Morse DE, Hansma PK. Bone indentation recovery time correlates with bond reforming time. Nature 2001;414 (6865):773-6.

[51] Fantner GE, Hassenkam T, Kindt JH, Weaver JC, Birkedal H, Pechenik L, et al. Sacrificial bonds and hidden length dissipate energy as mineralized fibrils separate during bone fracture. Nat Mater 2005;4(8):612-6.

[52] Hansma PK, Fantner GE, Kindt JH, Thurner PJ, Schitter G, Turner PJ, et al. Sacrificial bonds in the interfibrillar matrix of bone. J Musculoskelet Neuronal Interact 2005;5(4):313-5.

[53] Fantner GE, Adams J, Turner P, Thurner PJ, Fisher LW, Hansma PK. Nanoscale ion mediated networks in bone: osteopontin can repeatedly dissipate large amounts of energy. Nano Lett 2007;7(8):2491-8.

[54] Fantner GE, Birkedal H, Kindt JH, Hassenkam T, Weaver JC, Cutroni JA, et al. Influence of the degradation of the organic matrix on the microscopic fracture behavior of trabecular bone. Bone 2004;35(5):1013-22.

[55] Hassenkam T, Fantner GE, Cutroni JA, Weaver JC, Morse DE, Hansma PK. Highresolution AFM imaging of intact and fractured trabecular bone. Bone 2004;35(1): 4-10. 
[56] Fantner GE, Rabinovych O, Schitter G, Thurner P, Kindt JH, Finch MM, et al. Hierarchical interconnections in the nano-composite material bone: fibrillar crosslinks resist fracture on several length scales. Compos Sci Technol 2006;66(9): 1205-11.

[57] Kindt JH, Thurner PJ, Lauer ME, Bosma BL, Schitter G, Fantner GE, et al. In situ observation of fluoride-ion-induced hydroxyapatite-collagen detachment on bone fracture surfaces by atomic force microscopy. Nanotechnology 2007;18(135012):1-8.

[58] Thurner PJ, Oroudjev E, Jungmann R, Kreutz C, Kindt JH, Schitter G, et al. Imaging of bone ultrastructure using atomic force microscopy. Modern Research and Educational Topics in Microscopy; 2007. p. 37-48. (Journal Article).

[59] Grant CA, Brockwell DJ, Radford SE, Thomson NH. Effects of hydration on the mechanical response of individual collagen fibrils. Appl Phys Lett 2008;92(233902): 1-3.

[60] Tai K, Dao M, Suresh S, Palazoglu A, Ortiz C. Nanoscale heterogeneity promotes energy dissipation in bone. Nat Mater 2007;6(6):454-62.

[61] Cueru L, sfarghiu AT, Bala Y, Depalle B, Berthier Y, Follet H. Mechanical and physicochemical multiscale analysis of cortical bone. Comput Methods Biomech Biomed Engin 2011;14(sup1):223-5.

[62] Minary-Jolandan M, Yu MF. Uncovering nanoscale electromechanical heterogeneity in the subfibrillar structure of collagen fibrils responsible for the piezoelectricity of bone. ACS Nano 2009;3(7):1859-63.

[63] Halperin C, Mutchnik S, Agronin A, Molotskii M, Urenski P, Salai M, et al. Piezoelectric effect in human bones studied in nanometer scale. Nano Lett 2004;4(7): 1253-6.

[64] Kelly GM, Kilpatrick JI, van Es MH, Weafer PP, Prendergast PJ, Jarvis SP. Bone cell elasticity and morphology changes during the cell cycle. J Biomech 2011;44(8):1484-90. 
Figure 1

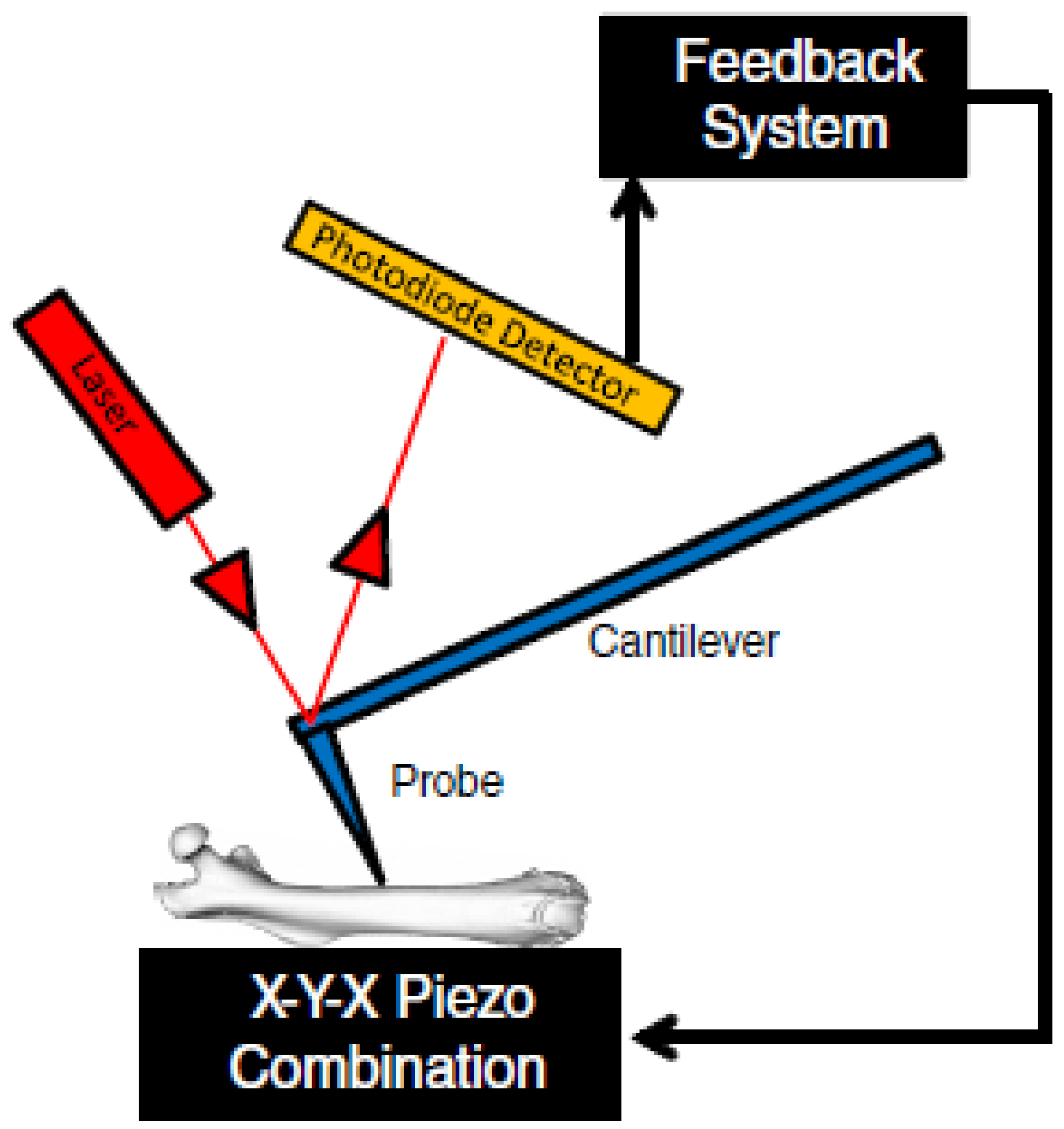

Fig. 1. Basic AFM operation. As a sample is raster-scanned under the probe in the $\mathrm{X}-\mathrm{Y}$ direction using piezoelectric actuators, the cantilever deflects up and down to accommodate the sample topography. The force of the interaction between the sample and the probe is most often measured by reflecting a laser off the back of the cantilever and into a detector. Since the cantilever acts like a linear spring, the force can be deter- mined from Hooke's Law $(\mathrm{F}=-\mathrm{kx}$ where $\mathrm{k}$ is the cantilever's spring constant and $\mathrm{x}$ is the deflection). A feedback control system is used to maintain a desired set point force between the probe and the sample by moving the sample up or down using a third piezoelectric actuator. This vertical movement generates a 3 dimensional height profile of the sample. 


\section{Figure 2}

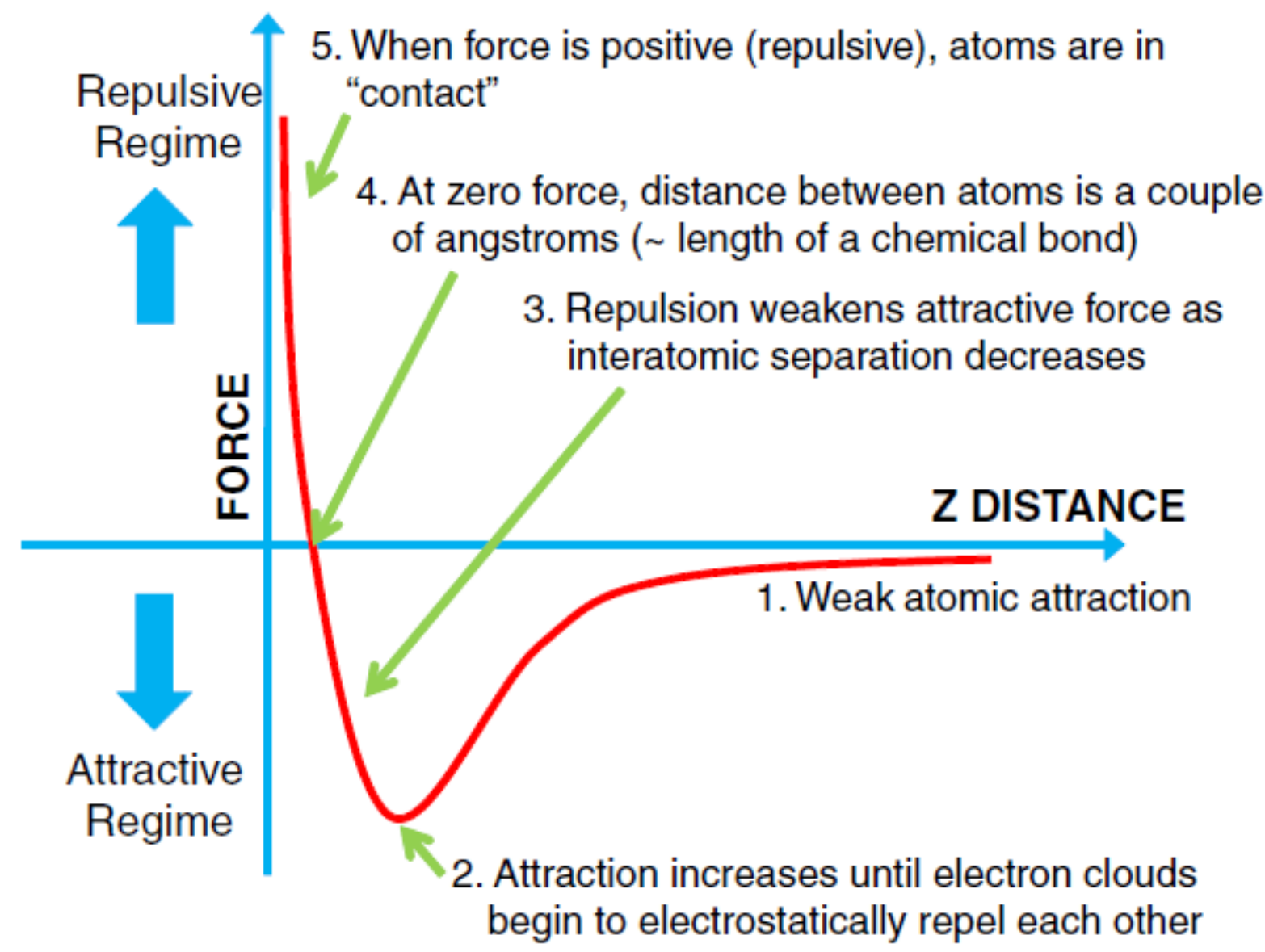

Fig. 2. van der Waals Potential Energy Curve. This curve describes the interactions occurring as the AFM probe is brought close to the sample's surface. At large distances, weak attractive interactions exist. As the distance decreases, this attractive force in- creases until the electron clouds of the atoms begin to interact and electrostatically repel each other. With further decrease in separation distance, this repulsive force weakens the overall attraction until a net force of zero is reached. The interactive forces then become positive (repulsive) and the atoms are said to be in "contact". 


\section{Figure 3}
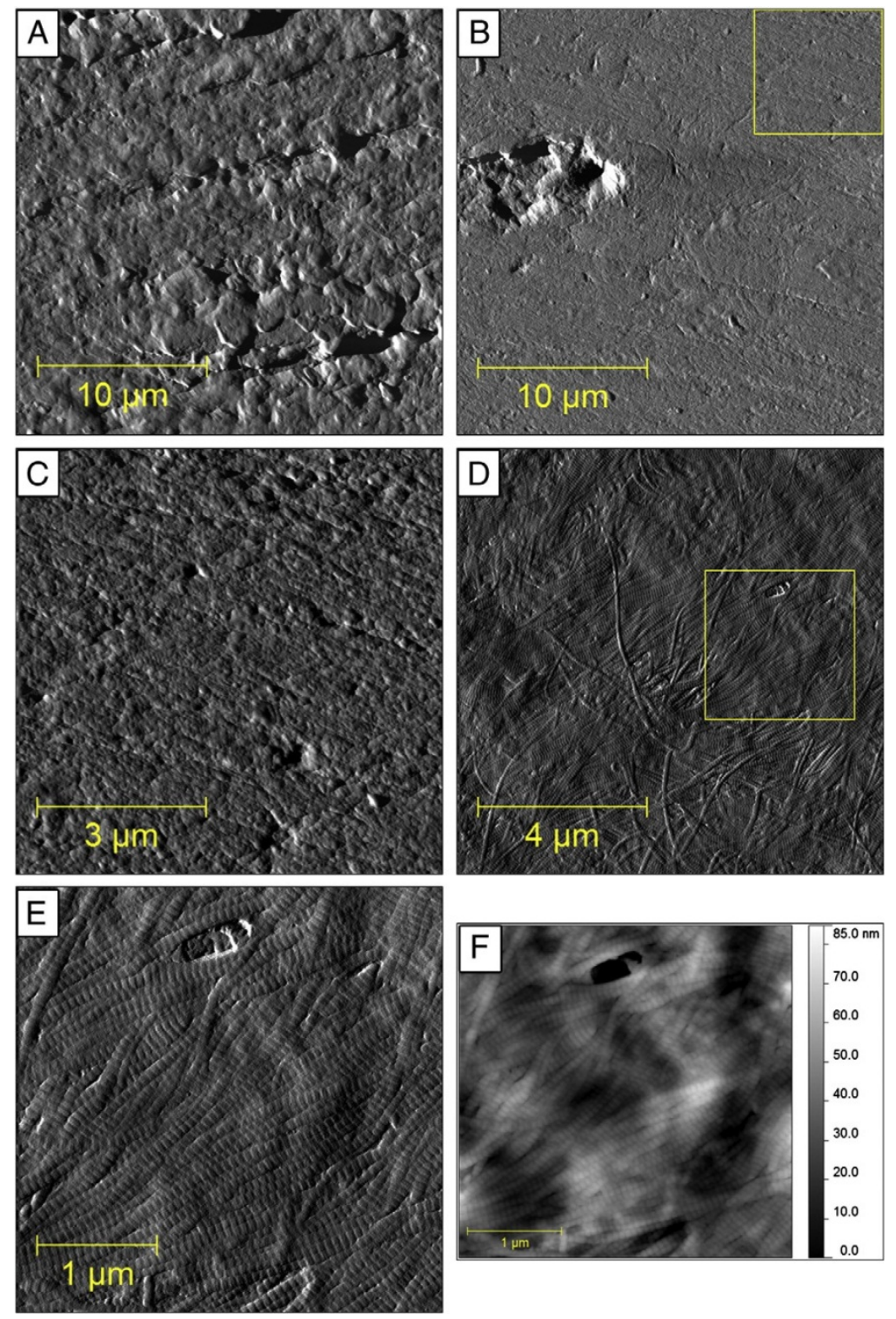

Fig. 3. AFM Imaging in Cortical Bone. These 6 panels are images from a murine bone, taken in peak force tapping mode. Panel A shows a $25 \mu \mathrm{m} 2$ region of the anterior surface of the bone prior to polishing or treatment, demonstrating a rough irregular surface. Panel B is another $25 \mu \mathrm{m} 2$ region on the same surface following polishing with a $3 \mu \mathrm{m}$ polycrystalline water-based diamond suspension to create a flat region of exposed intracortical bone. The square in panel B represents the $7.5 \mu \mathrm{m} 2$ location at which panel $\mathrm{C}$ was acquired. Panel $\mathrm{C}$ shows a blanket of mineral plates covering the underlying collagen. Panel $\mathrm{D}$ is a $10 \mu \mathrm{m} 2$ region of the same sample following treatment with EDTA as previously described [49]. With EDTA treatment, the rich collagen structure underlying the mineral is exposed. The square in panel $D$ represents the $3.5 \mu \mathrm{m} 2$ location at which panels $E$ and $F$ were acquired. Panels A-E represent the peak force error rather than the height. Error images are the instantaneous signal error at each pixel location, and often show finer surface characteristics than the height image. As a means of comparison, Panel F is the height image corresponding to the image in Panel E. The scale on the right side of Panel F shows that the color gradation represents a total height of $85 \mathrm{~nm}$. 


\section{Figure 4}

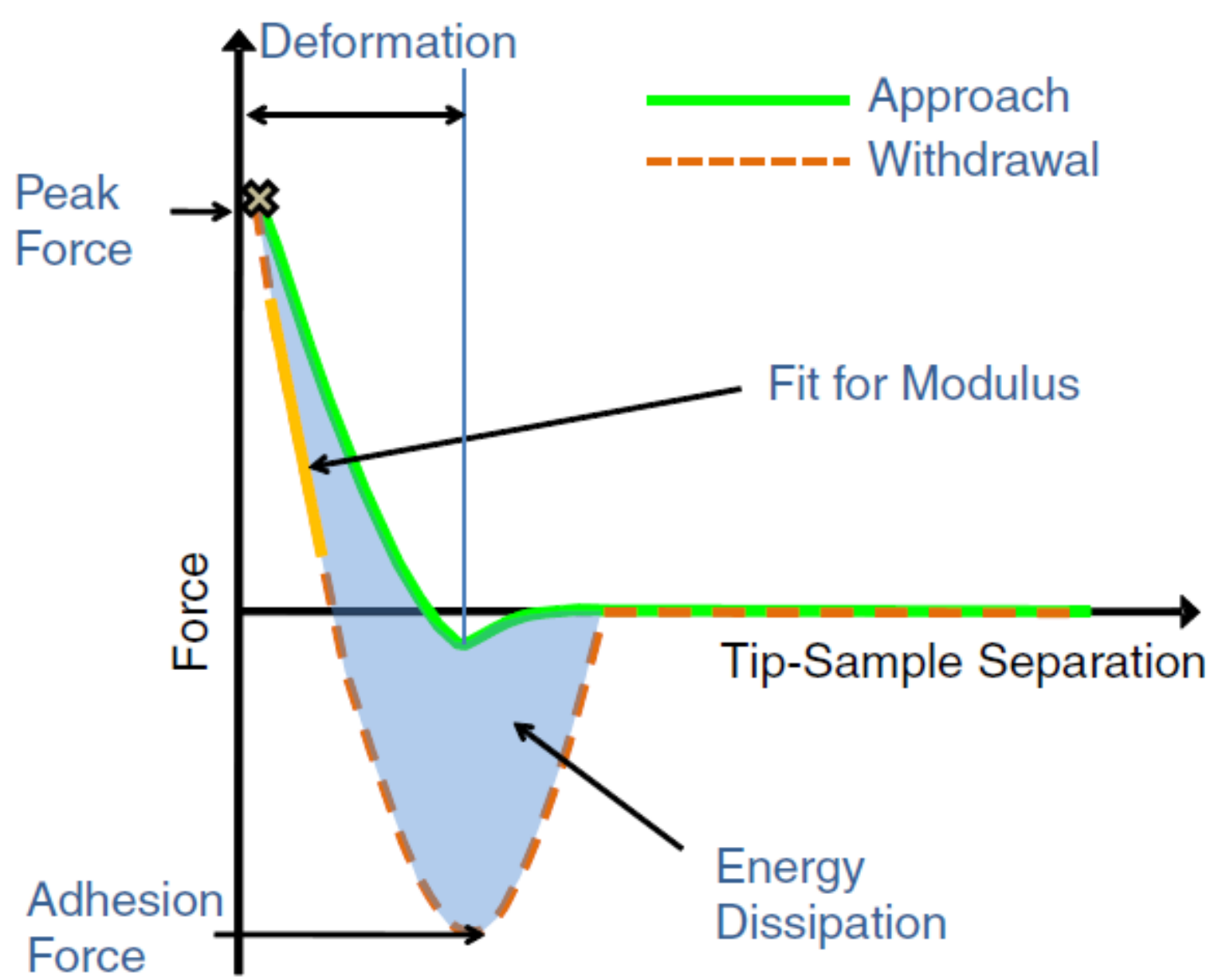

Fig. 4. AFM Indentation Force Curve. This curve describes the interaction as the AFM probe is pushed into the surface to a known load or displacement. From the raw curve, maximum (peak) force, deformation, adhesion force and energy dissipation (area between the approach and withdrawal curve) can be extracted. Using one of many contact mechanics models, the elastic indentation modulus of the sample can be calculated using curve fit analysis on the withdrawal portion of the force curve. The Hertz model is most common: $E=\left[3 F\left(1-v^{2}\right)\right] /\left(4 r^{\frac{1}{2}} \delta^{\frac{3}{2}}\right)$ where $\mathrm{F}$ is the applied load, $v$ is Poisson's ratio for the sample, $r$ is the radius of curvature of the AFM probe and $\delta$ is the indentation depth. 
Figure 5

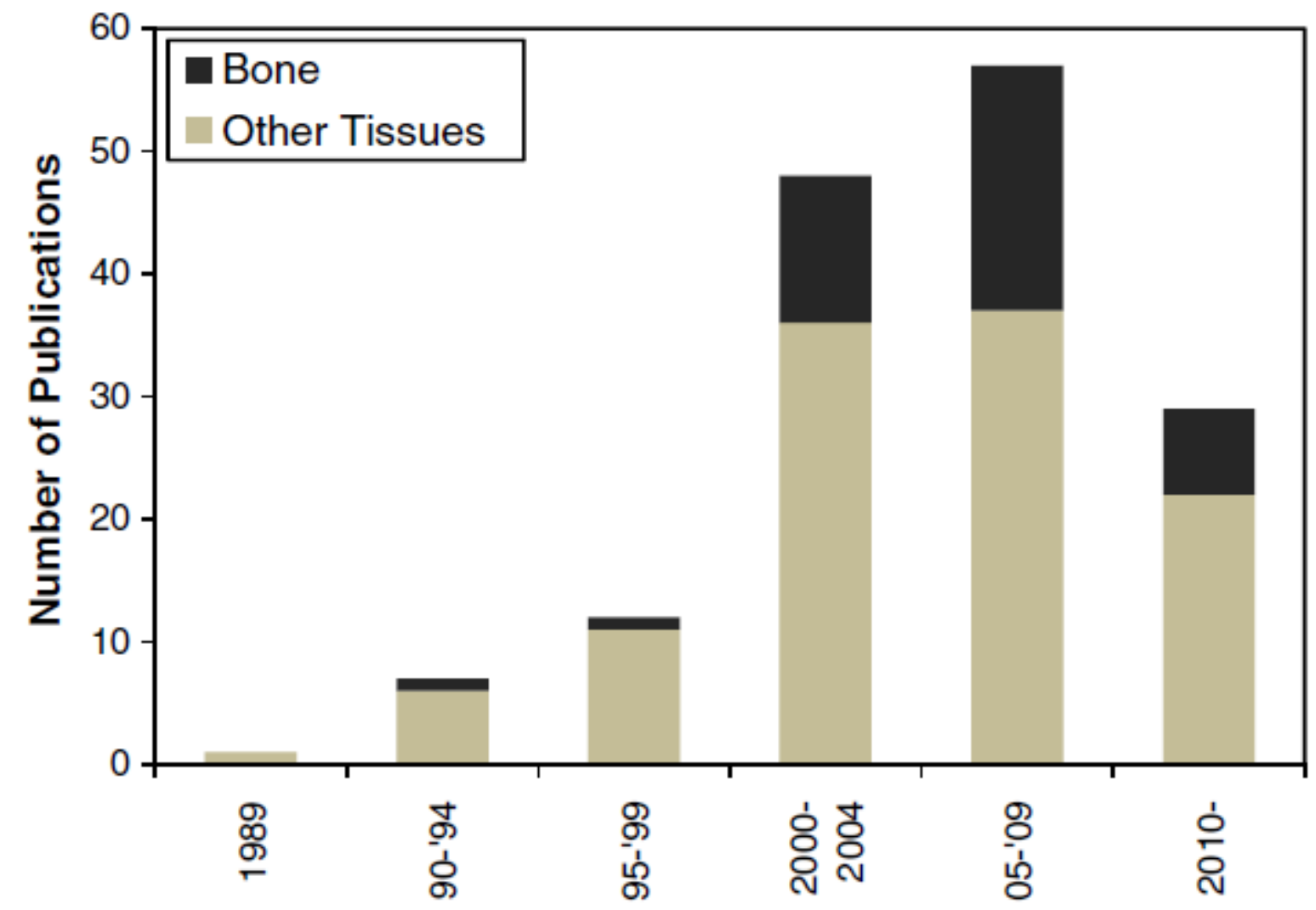

Fig. 5. Studies of Collagen-Based Tissues using Scanning Probe Microscopy (SPM). SPM includes both Scanning Tunnelling Microscopy (STM) and Atomic Force Microscopy (AFM). All studies in the figure were performed using AFM with the exception of one in 1989 and one in 1991. There has been a steady rise in AFM use in collagen-based tis- sues over the past 22 year, but the majority of these studies ( 75\%) were in tissue other than bone. 